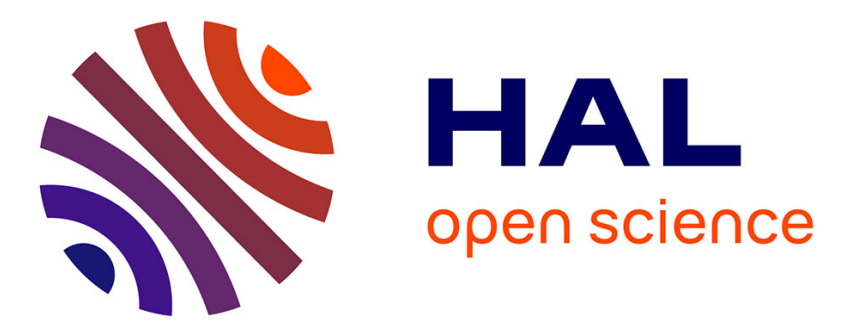

\title{
Spatio-Spectral Multichannel Reconstruction from few Low-Resolution Multispectral Data
}

Mohamed El Amine Hadj-Youcef, François Orieux, Aurélia Fraysse, A.

Abergel

\section{- To cite this version:}

Mohamed El Amine Hadj-Youcef, François Orieux, Aurélia Fraysse, A. Abergel. Spatio-Spectral Multichannel Reconstruction from few Low-Resolution Multispectral Data. 26th European Signal Processing Conference (EUSIPCO 2018), Sep 2018, Rome, Italy. hal-01952286

\section{HAL Id: hal-01952286 \\ https://hal-centralesupelec.archives-ouvertes.fr/hal-01952286}

Submitted on 12 Dec 2018

HAL is a multi-disciplinary open access archive for the deposit and dissemination of scientific research documents, whether they are published or not. The documents may come from teaching and research institutions in France or abroad, or from public or private research centers.
L'archive ouverte pluridisciplinaire HAL, est destinée au dépôt et à la diffusion de documents scientifiques de niveau recherche, publiés ou non, émanant des établissements d'enseignement et de recherche français ou étrangers, des laboratoires publics ou privés. 


\title{
Spatio-Spectral Multichannel Reconstruction from few Low-Resolution Multispectral Data
}

\author{
M.A. Hadj-Youcef*†, F. Orieux* ${ }^{* \dagger}$ A. Fraysse*, A. Abergel ${ }^{\dagger}$ \\ * Laboratoire des Signaux et Systèmes, Univ. Paris-Sud, CNRS, CentraleSupélec, Université Paris-Saclay \\ 3 rue Joliot-Curie, 91192 Gif-sur-Yvette, France \\ Email: amine.hadjyoucef@ias.u-psud.fr \\ † Institut d'Astrophysique Spatiale, CNRS, UMR 8617, Univ. Paris-Sud, Université Paris-Saclay \\ Univ. Paris-Sud, 91405 Orsay, France
}

\begin{abstract}
This paper deals with the reconstruction of a 3-D spatio-spectral object observed by a multispectral imaging system, where the original object is blurred with a spectralvariant PSF (Point Spread Function) and integrated over few broad spectral bands. In order to tackle this ill-posed problem, we propose a multispectral forward model that accounts for direct (or auto) channels and between (or cross) channels degradation, by modeling the imaging system response and the spectral distribution of the object with a piecewise linear function. Reconstruction based on regularization methods is proposed, by enforcing spatial and spectral smoothness of the object. We test our approach on simulated data of the Mid-InfraRed Instrument (MIRI) Imager of the James Webb Space Telescope (JWST). Results on simulated multispectral data show a significant improvement over the conventional multichannel method.
\end{abstract}

Index Terms - Inverse problems, Image reconstruction, Deconvolution, System modeling, Multispectral restoration

\section{INTRODUCTION}

Multispectral imaging systems are used in many fields, e.g. astrophysics [1], remote sensing [2], medicine [3] or microscopy [4]. This paper deals with the inverse problem of joint restoration. Our goal is to reconstruct a discrete 3D spatio-spectral object from a small number of 2-D MultiSpectral (MS) observed data when this continuous 3-D object is degraded by the instrument that suffers the diffraction due to the limited size of the optical system. This physical degradation affects its spatial resolution (in the form of blur) accordingly to the wavelength. Moreover, before its spatial sampling, the blurred object is integrated by the detector over the different wide spectral bands, which results in low spectral resolution multispectral data. Therefore, the multispectral data are severely degraded and contain limited spectral information about the original object.

Multichannel restoration has been extensively studied in the literature. Multichannel forward models have been proposed in [5], [6], where the system response is a block-diagonal matrix with circulant blocks. For instance, [7], [8], [9] address multichannel 2-D deconvolution problem for hyperspectral image deconvolution. They take into account the within-channel degradation, but not the between channel (or cross-channel) degradation. Hence, this approach is not suitable for MS imaging, especially if spectral bands are broad and overlapping, which implies a strong correlation between channel. In [10] a model is proposed that reduces these limitations since the system response is represented by a block matrix corresponding to within and between channel degradation. However, this model is mostly used when the number of channels and observations is the same, e.g. color image restoration [11], [12], [13].

In this paper we propose a multispectral forward model that accounts for within- and between-spectral channel degradations (or auto and cross-channel), where (1) the number of MS data is much lower than the number of spectral channels and (2) a set of low-resolution MS data are degraded by a spectral-variant PSF and integrated over broad spectral bands. Reconstruction of a spatio-spectral object is performed using regularization, by accounting for spatial and spectral quadratic regularization. Simulated results are provided with a comparison to multichannel 2-D deconvolution for an application to the MIRI Imager on board the $\mathrm{JWST}^{1}$.

This paper is organized as follows. In Sec. II we present the problem formulation. The imaging system response and the forward model are described in Sec. III. The reconstruction method is presented in Sec. IV. Simulation and results are presented in Sec. V including a brief description of the JWST/MIRI Imager. Conclusions and perspectives are in Sec. VI.

\section{PROBlem FORMUlation}

The general form of the multispectral problem we are considering is the one proposed in [10], [11]. It follows the following discrete linear forward model :

$$
\boldsymbol{y}=\boldsymbol{H} \boldsymbol{x}+\boldsymbol{n},
$$

where $\boldsymbol{x}=\left[\boldsymbol{x}^{(1) T}, \boldsymbol{x}^{(2) T}, \ldots, \boldsymbol{x}^{(M) T}\right]^{T}$ is the stack of $M$ spectral channels represented in a vector form, each channel contains $N \times N$ pixels, where $x_{i, j}^{(m)}$ denotes the $(i, j)^{t h}$ spatial position in the $(m)^{t h}$ wavelength. The vector $\boldsymbol{y}=$ $\left[\boldsymbol{y}^{(1) T}, \boldsymbol{y}^{(2) T}, \ldots, \boldsymbol{y}^{(P) T}\right]^{T} \in \mathbb{R}^{P N^{2}}$ is the stack of multispectral observed data acquired via $P$ broad spectral bands of the imaging system. $\boldsymbol{n}=\left[\boldsymbol{n}^{(1) T}, \boldsymbol{n}^{(2) T}, \ldots, \boldsymbol{n}^{(P) T}\right]^{T} \in \mathbb{R}^{P N^{2}}$

\footnotetext{
${ }^{1}$ https://jwst.nasa.gov/
} 
represents an additive unknown noise. The full system response is a $P N^{2}$ by $M N^{2}$ block matrix,

$$
\boldsymbol{H}=\left[\begin{array}{cccc}
\boldsymbol{H}^{1,1} & \boldsymbol{H}^{1,2} & \cdots & \boldsymbol{H}^{1, M} \\
\vdots & \vdots & \ddots & \vdots \\
\boldsymbol{H}^{P, 1} & \boldsymbol{H}^{P, 2} & \cdots & \boldsymbol{H}^{P, M}
\end{array}\right], \quad P \ll M,
$$

defined by a set of $P \times M$ Toeplitz sub-matrices $\boldsymbol{H}^{p, m} \in$ $\mathbb{R}^{N^{2} \times N^{2}}$, which are approximated for computational ease by circulant blocks. All sub-matrices are ill-conditioned, meaning that $\boldsymbol{H}$ is also ill-conditioned, which leads to an ill-posed problem. Each multispectral observation $\boldsymbol{y}^{(p)}$ depends on all spectral channels through the blocks of $\boldsymbol{H}$, where $\boldsymbol{H}^{p, t}, t=m$ represents the direct (or auto) observation, whereas the block $\boldsymbol{H}^{p, t}, t \neq m$ accounts for between (or cross) degradation occurring between channels. We are interested in the particular case where we have few low-resolution multispectral observed data compared to spectral channels, i.e. $P \ll M$, which means there is a lack of spectral information in the data. For instance, multispectral data observed by the JWST/MIRI imager, e.g. $P=9$ and $M=1000$, and the size of $\boldsymbol{H}$ is $9 \times 256^{2}$ by $1000 \times 256^{2}$, for a $256 \times 256$ pixel detector.

\section{MODELING OF WITHIN AND BETWEEN CHANNELS DEGRADATION}

\section{A. Object Model}

We first define the 3-D spatio-spectral object of interest with $\phi(\alpha, \beta, \lambda): \mathbb{R}^{3} \rightarrow \mathbb{R}$, having two spatial parameters $(\alpha, \beta) \in \mathbb{R}^{2}$ and one spectral parameter $\lambda \in \mathbb{R}_{+}$. In order to handle the lack of spectral information in the data, we propose to perform approximation of the $M$ spectral channels by $M^{\prime}$ channels, with $M^{\prime}<M$, by modeling the object spectral distribution with a piecewise linear function [14]. Moreover, we are interested in reconstructing a discrete version of the object, hence, we define two basis functions, for spatial and spectral discretizations, $b_{s}$ and $b_{\lambda}$, respectively. They are defined upon two grids, $\mathcal{G}_{s}=\left\{\alpha_{k}, \beta_{l}\right\}_{k, l=1}^{N}$ and $\mathcal{G}_{\lambda}=\left\{\lambda^{(m)}\right\}_{m=1}^{M^{\prime}}$, respectively. The object is modeled by

$$
\phi(\alpha, \beta, \lambda)=\sum_{m=1}^{M^{\prime}} \sum_{k, l=1}^{N} x_{k, l}^{(m)} b_{s}^{(k, l)}(\alpha, \beta) b_{\lambda}^{(m)}(\lambda),
$$

where $b_{\lambda}^{(m)}(\lambda)$ is a uniform piecewise linear function, for instance first-order B-spline function [15]. The parameter $M^{\prime}$ compromises between the sharpness of the spectral sampling of the modeled object and the unknown spectral channels to reconstruct $x^{(m)}$.

\section{B. Imaging System Response}

In this section we provide the multispectral imaging system response by establishing an imaging system model that relates the input to the output. The instrument we are considering is composed of an optical system and a detector. Due to light diffraction of $\phi$, the optical system response is modeled by a 2-D spatial convolution [16] with a spectral variant optical response, known as Point Spread Function (PSF) $h(\alpha, \beta, \lambda)$.
This blurs the object accordingly to the wavelength and limits its spatial resolution (as illustrated in Sec. V). The blurred object is integrated over broad spectral bands $\tau_{p}(\lambda)$ and sampled pixel-by-pixel on the 2-D detector grid, $\mathcal{G}_{\text {samp }}=$ $\left\{\alpha_{i}, \beta_{j}\right\}_{i, j=1}^{N}: \alpha_{i}, \beta_{j}$ being the 2-D angular positions of pixels $(i, j)$ and $N$ is the total number of pixel according dimensions $\alpha$ and $\beta$. We introduce a basis function $b_{\text {samp }}^{(i, j)}(\alpha, \beta)$ to carry out spatial sampling. It is defined on the pixel sensitive surface $\Omega_{\text {pix }}$. Moreover, a noise term $n_{i, j}^{(p)}$ is added for each pixel $(i, j)$ and band $p$, e.g. readout noise of the detector. Finally, the imaging system model is given by

$$
\begin{aligned}
& y_{i, j}^{(p)}=\int_{\mathbb{R}_{+}} \tau_{p}(\lambda)\left(\int \int _ { \Omega _ { \mathrm { pix } } } \left(\iint_{\mathbb{R}^{2}} \phi\left(\alpha^{\prime}, \beta^{\prime}, \lambda\right)\right.\right. \\
& \left.\left.h\left(\alpha-\alpha^{\prime}, \beta-\beta^{\prime}, \lambda\right) d \alpha^{\prime} d \beta^{\prime}\right) b_{\mathrm{samp}}^{(i, j)}(\alpha, \beta) d \alpha d \beta\right) d \lambda+n_{i, j}^{(p)}
\end{aligned}
$$

this model links the 3-D continuous input $\phi(\alpha, \beta, \lambda)$ to the 2-D discrete output $y_{i, j}^{(p)}$ through a complex instrument response, which includes spectral windowing and five sums, two for spatial 2-D convolutions, two for spatial sums and one for spectral integration. Note that the above model does not include any non-ideal characteristics of the detector, which are assumed to be corrected upstream.

\section{Forward Model and definition of $\boldsymbol{H}^{p, m}$}

The discrete forward model links the discrete spectral channels to the discrete multispectral data. It is obtained by substituting equation (3) in (4). This yields

$$
y_{i, j}^{(p)}=\sum_{m=1}^{M^{\prime}} \sum_{k, l=1}^{N} H_{i, j ; k, l}^{p, m} x_{k, l}^{(m)}+n_{i, j}^{(p)},
$$

with

$$
\begin{gathered}
H_{i, j ; k, l}^{p, m}=\int_{\mathbb{R}_{+}} \tau_{p}(\lambda) b_{\lambda}^{(m)}(\lambda)\left(\int \int _ { \Omega _ { \mathrm { pix } } } \left(\iint_{\mathbb{R}^{2}} b_{s}^{(k, l)}\left(\alpha^{\prime}, \beta^{\prime}\right)\right.\right. \\
\left.\left.h\left(\alpha-\alpha^{\prime}, \beta-\beta^{\prime}, \lambda\right) d \alpha^{\prime} d \beta^{\prime}\right) b_{\mathrm{samp}}^{(i, j)}(\alpha, \beta) d \alpha d \beta\right) d \lambda .
\end{gathered}
$$

In addition, we consider for instance a rectangular impulse function [17] for the sampling function. i.e. $b_{\mathrm{samp}}^{(i, j)}(\alpha, \beta)=$ $\frac{1}{\triangle \alpha \triangle \beta} \Pi_{\triangle \alpha, \triangle \beta}\left(\alpha-\alpha_{i}, \beta-\beta_{j}\right)$, with $\triangle \alpha, \triangle \beta$ are the sampling steps according to dimensions $\alpha$ and $\beta$, respectively. Thus, the system response becomes a convolution matrix $H_{i, j ; k, l}^{p, m}=$ $H_{i-k ; j-l}^{p, m}$. Therefore, the vector-matrix representation of $(5)$ is

$$
\boldsymbol{y}^{(p)}=\sum_{m=1}^{M^{\prime}} \boldsymbol{H}^{p, m} \boldsymbol{x}^{(m)}+\boldsymbol{n}^{(p)},
$$

where the $p$-th multispectral data $\boldsymbol{y}^{(p)}$ is a sum of $M^{\prime}$ discrete 2-D spatial convolutions between spectral channels and convolution matrices $\boldsymbol{H}^{p, m}$ (blocks of the matrix $\boldsymbol{H}$ in (2)). Thus, it accounts for within and between channels degradation. The discrete multispectral forward model with the full imaging system $\boldsymbol{H}$ response takes the form in (1). 


\section{RECONSTRUCTION}

Reconstruction of the object of interest $\phi$ relies on the reconstruction of its spectral channels $\boldsymbol{x}^{(m)}: m=1,2, \ldots, M^{\prime}$ using a regularization method. The solution of reconstruction is obtained by minimizing an objective function $\mathcal{J}(\boldsymbol{x})$,

$$
\hat{\boldsymbol{x}}=\underset{\boldsymbol{x}^{(1)}, \ldots, \boldsymbol{x}^{\left(M^{\prime}\right)}}{\operatorname{argmin}}\{\mathcal{J}(\boldsymbol{x})\},
$$

this method is called regularized least squares [18], where we add regularization terms to $\mathcal{J}(\boldsymbol{x})$ in order to correct the illposedness of the problem. The objective function is defined by

$$
\mathcal{J}(\boldsymbol{x})=\mathcal{Q}(\boldsymbol{x}, \boldsymbol{y})+\mathcal{R}_{s}(\boldsymbol{x})+\mathcal{R}_{\lambda}(\boldsymbol{x}) .
$$

where the first term $\mathcal{Q}(\boldsymbol{x}, \boldsymbol{y})=\|\boldsymbol{y}-\boldsymbol{H} \boldsymbol{x}\|_{2}^{2}$ is the data fidelity. It enforces agreement of the solution with the data. The second term $\mathcal{R}_{s}(\boldsymbol{x})=\mu_{s}\left\|\boldsymbol{D}_{s} \boldsymbol{x}\right\|_{2}^{2}$ is a spatial regularization. It enforces spatial smoothness between neighboring pixels of $\boldsymbol{x}$, with $\boldsymbol{D}_{s} \in \mathbb{R}^{M^{\prime} N^{2} \times M^{\prime} N^{2}}$ is a second-order finite difference operator along the spatial dimension. The last term refers to spectral regularization $\mathcal{R}_{\lambda}(\boldsymbol{x})=\mu_{\lambda}\left\|\boldsymbol{D}_{\lambda} \boldsymbol{x}\right\|_{2}^{2}$. It enforces the similarity between intensity values of corresponding pixels in neighboring channels, with $\boldsymbol{D}_{\lambda} \in \mathbb{R}^{M^{\prime} N^{2} \times M^{\prime} N^{2}}$ is a firstorder finite difference operator along the spectral direction. $\mu_{s}$ and $\mu_{\lambda}$ are regularization parameters, they are set to compromise between fidelity to the data and spatial smoothness, and spectral smoothness across channel, respectively.

By rewriting the objective function, (9) becomes

$$
\mathcal{J}(\boldsymbol{x})=\|\boldsymbol{y}-\boldsymbol{H} \boldsymbol{x}\|_{2}^{2}+\mu_{s}\left\|\boldsymbol{D}_{s} \boldsymbol{x}\right\|_{2}^{2}+\mu_{\lambda}\left\|\boldsymbol{D}_{\lambda} \boldsymbol{x}\right\|_{2}^{2} .
$$

It is the sum of quadratic terms only, therefore, an explicit solution is available by canceling the gradient of $\mathcal{J}(\boldsymbol{x})$. This yields

$$
\boldsymbol{Q} \hat{\boldsymbol{x}}=\boldsymbol{H}^{T} \boldsymbol{y},
$$

with $\boldsymbol{Q}=\left(\boldsymbol{H}^{T} \boldsymbol{H}+\boldsymbol{D}_{s}^{T} \boldsymbol{D}_{s}+\boldsymbol{D}_{\lambda}^{T} \boldsymbol{D}_{\lambda}\right)$. It contains Toepltiz blocks $\boldsymbol{Q}^{(i, j)}: i, j=1, \ldots, M$ of size $N^{2} \times N^{2}$. However, $\boldsymbol{Q}^{(i, j)} \neq \boldsymbol{Q}^{(i+t, j+t)}$, hence $\boldsymbol{Q}$ is not a Toeplitz matrix. We propose to compute the solution without inverting $Q$, but by computing the solution iteratively using the following form:

$$
\hat{\boldsymbol{x}}^{k+1}=\hat{\boldsymbol{x}}^{k}-a\left[\boldsymbol{Q} \hat{\boldsymbol{x}}^{k}-\boldsymbol{H}^{T} \boldsymbol{y}\right],
$$

with $\hat{\boldsymbol{x}}^{k=0}=\mathbf{0}$ corresponds to the initialization and $a$ is a convergence parameter of the algorithm. A conjugated gradient (CG) algorithm [19] is implemented.

\section{Simulation Results}

\section{A. JWST/MIRI Imager}

We apply the proposed reconstruction algorithm to multispectral data simulated using the model in (4) of the MidInfraRed Instrument (MIRI) Imager [20] on-board the James Webb Space Telescope (JWST), the next flagship space telescope of NASA, ESA and the Canadian Space Agency (CSA) to be launched in 2020. The imager provides nine multispectral observations $(P=9)$ integrated over a broad range of spectral bands, from $5 \mu \mathrm{m}$ to $28 \mu \mathrm{m}$ [21]. The nine bands are shown in Fig. 1. Note that overlapping of the spectral bands increase the between channels degradation. The MIRI Imager detector has a pixel pitch of 0.11 arcsecond, i.e. $\Omega_{\text {pix }}=0.11 \times 0.11$ $\operatorname{arcsecond}^{2}$. We use the official PSF simulator of the JWST mission, WebbPSF [22], [23], to simulate a realistic PSF image at different wavelengths, as shown in Fig. 2. The PSF is complex due to the segmented mirror of the JWST. We clearly observe an enlargement of the PSF according to the wavelength, i.e. the longer the wavelength the wider the PSF, as expected from diffraction theory [16].

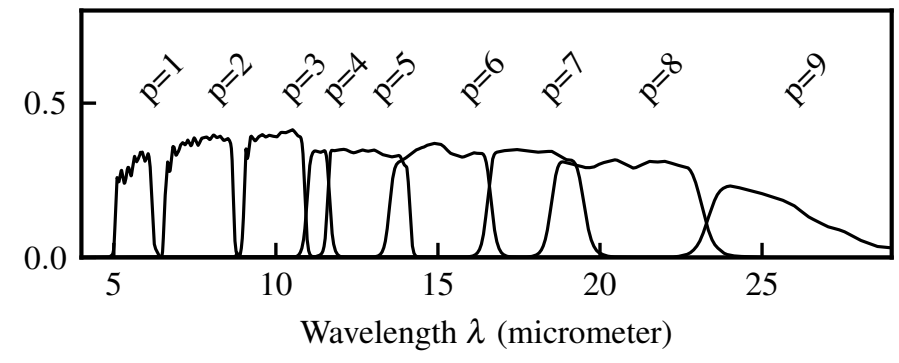

Fig. 1. The nine broad bands of the JWST/MIRI Imager [21] covering the mid-infrared wavelength range from 5 to $28 \mu \mathrm{m}$.
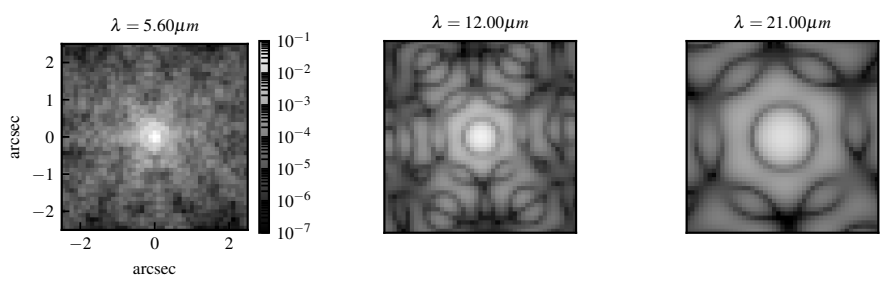

Fig. 2. Monochromatic PSF of the JWST/MIRI imager simulated using WebbPSF [22], [23] and displayed in the same logarithmic scale. We clearly observe the dependency of the PSF to the wavelength as expected from diffraction theory.

\section{B. Setup of the Experiment}

The original 3D object $\phi$ is a simplified spatio-spectral model of the Horse Head nebula [24]. A spatial region of $256 \times 256$ pixels $(N=256)$ is taken for the simulation with $M=1000$ spectral samples uniformly distributed within 4-28 $\mu \mathrm{m}$. Nine multispectral data are simulated using (4) with a zero-mean white Gaussian noise added in order to obtain a global Signal-to-Noise Ratio (SNR) of 30, 20, $10 \mathrm{~dB}$.

$$
S N R_{(d B)}=10 \log _{10}\left(\frac{\frac{1}{P N^{2}}\|\boldsymbol{y}\|_{2}^{2}}{\sigma_{n}^{2}}\right),
$$

where $\sigma_{n}$ is the standard deviation of the noise. $P$ is the number of MS data and $N^{2}$ is the total number of pixels in the MS data.

Reconstruction results are summarized in Table I together with a comparison between the proposed method and the multichannel 2-D deconvolution method (MDec) [6] (independent channel restoration using an averaged PSF per channel). The regularization parameters $\mu_{s}$ and $\mu_{\lambda}$ are adjusted by 
running the code for different values in a range $\left[10^{-4}, 10^{-2}\right]$ and keeping the pair that minimizes the objective function $\mathcal{J}\left(\hat{\boldsymbol{x}}\left(\hat{\mu_{s}}, \hat{\mu_{\lambda}}\right)\right)$. For a quantitative comparison between the original object $f_{\text {orig }}$ and the reconstructed $f_{\text {rec }}$, we compute the relative reconstruction error as defined by

$$
\operatorname{Error}(\%)=100 \times\left\|\boldsymbol{f}_{\text {orig }}-\boldsymbol{f}_{\text {rec }}\right\|_{2} /\left\|\boldsymbol{f}_{\text {orig }}\right\|_{2} .
$$

\section{Discussion}

Fig. 3 shows the spectral reconstruction result of one single pixel $(100,150)$, comparing the original spectrum $\phi_{\text {orig }}$, the reconstructed spectrum using our method $\phi_{\text {rec }}$, and the reconstructed spectrum using multichannel 2-D deconvolution $\phi_{M D e c}$. The original spectral distribution is complex with spectral features at short wavelengths $(4-13 \mu \mathrm{m})$ and continuum. Therefore, an accurate reconstruction using a few MS data is difficult, if not impossible, without using a strong prior knowledge of the spectrum of the object. The reconstructed spectrum computed with our method (using piecewise linear model) allows us to reconstruct an envelope-like spectral distribution which significantly increases the spectral resolution compared to multichannel 2-D deconvolution. Several values of $M^{\prime}=\{20,40,60\}$ have been tested, and the reconstruction results for three wavelengths, 7.8, 16 and $21 \mu \mathrm{m}$, are reported in Table I. Increasing $M^{\prime}$ improves the spectral resolution of the object model, but increases the between channels degradation and the number of unknowns. Moreover, we find that there is not much error improvement for $M^{\prime}>60$. In any case, the proposed reconstruction shows smaller reconstruction errors compared to the multichannel 2-D deconvolution ; this is due to our model accounting for within and between channels degradation. Spatial reconstruction results at different wavelengths are illustrated in Fig. 4. As anticipated, a better reconstruction is obtained at $\lambda=16 \mu \mathrm{m}$ and $\lambda=21 \mu \mathrm{m}$ than at $\lambda=7.8 \mu \mathrm{m}$ (see the fourth row of the figure) since within the integration windows at long wavelengths the spectrum of the object does not contain any feature.

TABLE I

Proposition AND COMPARISON OF Horse HeAd NEBUla [24] OF SIZE $1000 \times 256 \times 256$

\begin{tabular}{|c|c|c|c|c|c|}
\hline \multirow[t]{2}{*}{$\begin{array}{l}\text { SNR } \\
(\mathrm{dB})\end{array}$} & \multirow[t]{2}{*}{$\begin{array}{c}\lambda \\
(\mu \mathrm{m})\end{array}$} & \multicolumn{3}{|c|}{$\begin{array}{c}\text { Error (\%) } \\
\text { Proposed Reconstruction }\end{array}$} & \multirow[t]{2}{*}{ MDec } \\
\hline & & $M^{\prime}=20$ & $\mathrm{M}^{\prime}=40$ & $M^{\prime}=60$ & \\
\hline \multirow{3}{*}{30} & 7,8 & 49,44 & 42,37 & 41,42 & 52,85 \\
\hline & 16,0 & 2,44 & 4,11 & 4,80 & 7,89 \\
\hline & 21,0 & 1,87 & 3,82 & 4,26 & 11,92 \\
\hline \multirow{3}{*}{20} & 7,8 & 49,50 & 43,07 & 41,46 & 52,84 \\
\hline & 16,0 & 7,41 & 7,66 & 8,98 & 8,02 \\
\hline & 21,0 & 4,42 & 5,40 & 5,77 & 11,97 \\
\hline \multirow{3}{*}{10} & 7,8 & 50,71 & 43,71 & 42,38 & 52,84 \\
\hline & 16,0 & 19,67 & 21,25 & 25,51 & 8,56 \\
\hline & 21,0 & 10,85 & 11,31 & 13,38 & 12,13 \\
\hline
\end{tabular}

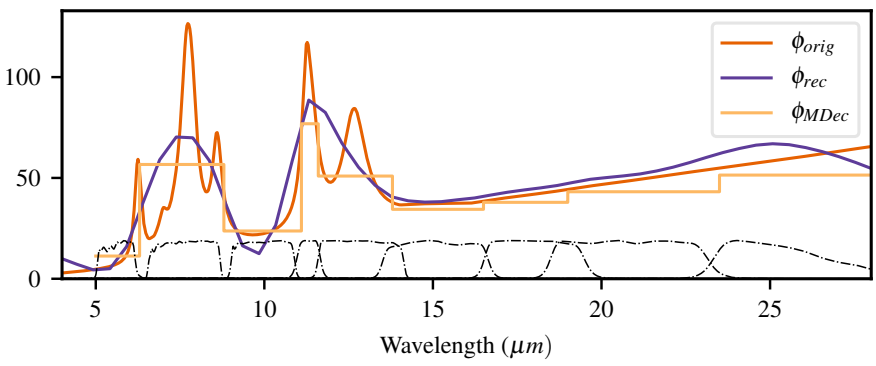

Fig. 3. Comparison between one single pixel $(100,150)$ spectrum from the original object $\phi_{\text {orig }}$, the proposed reconstruction $\phi_{\text {rec }}\left(\right.$ with $\left.M^{\prime}=60\right)$ and the multichannel 2-D deconvolution $\phi_{M D e c}$. The nine MS data $(P=9)$ were corrupted with zero-mean Gaussian noise of $30 \mathrm{~dB}$.
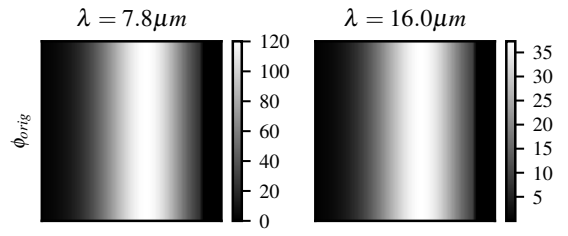

$\lambda=21.0 \mu m$

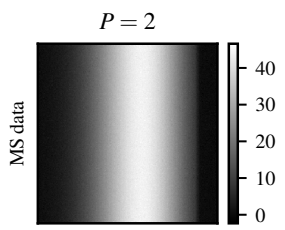

$P=6$

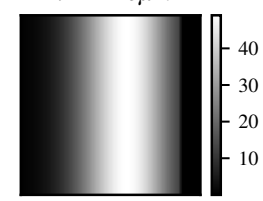

$P=8$
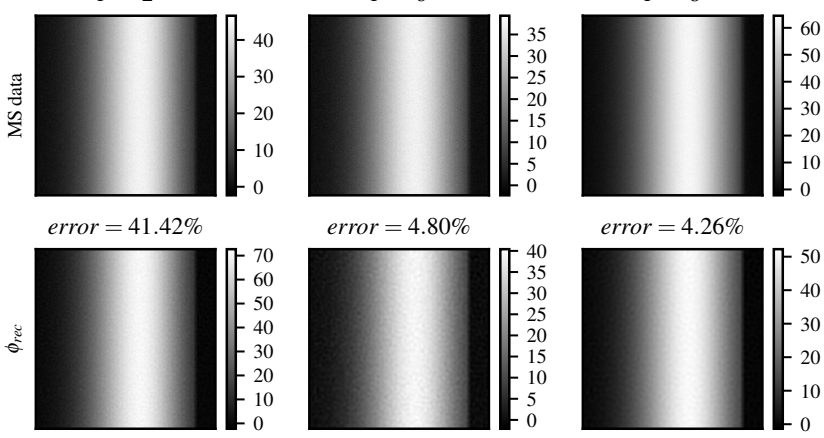

error $=4.80 \%$

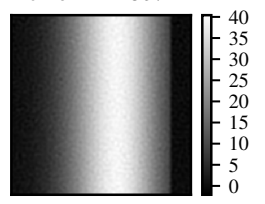

error $=4.26 \%$
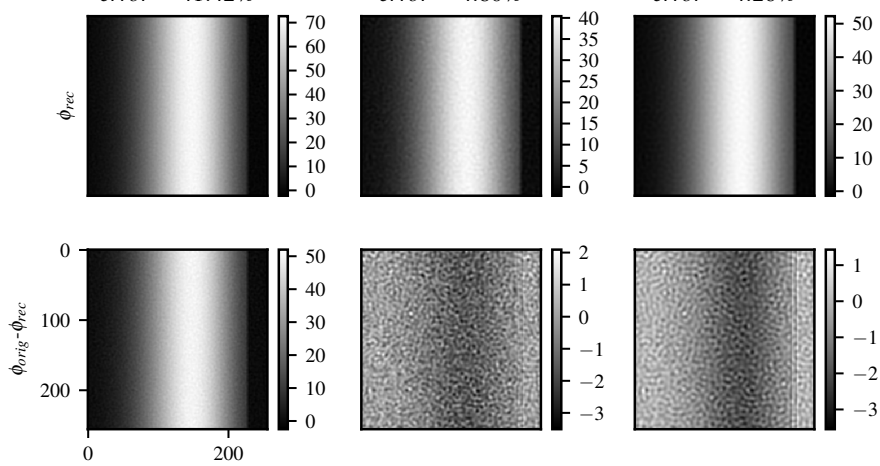

Fig. 4. [First row] Original spectral channel of the Horse Head nebula. [Second row] Simulated MS data with $30 \mathrm{~dB}$ corresponding to the bands that include wavelengths of the first row (see Fig. 1). [Third row] Proposed reconstruction. [Fourth row] Difference between the original and reconstructed spectral channels. The original and reconstructed objects are in physical units, whereas MS data are in detector units.

\section{CONCLUSION}

In this paper we address the reconstruction of 3-D spatiospectral object observed by a multispectral imaging system from a few low-resolution data. A discrete forward model is defined accounting for within and between channels degradation using a piecewise linear function to model the spectral distribution of the sought object. A quadratic reconstruction is proposed by considering spatial and spectral regularization terms. Results on simulated data applied to the JWST/MIRI Imager highlights the complexity of the problem. A clear increase of spatial and spectral distribution is achieved compared to multichannel 2-D deconvolution method. 


\section{REFERENCES}

[1] Roberto Lionello, Jon A Linker, and Zoran Mikić, "Multispectral emission of the sun during the first whole sun month: Magnetohydrodynamic simulations," The Astrophysical Journal, vol. 690, no. 1, pp. 902, 2008.

[2] David A Landgrebe, Signal theory methods in multispectral remote sensing, vol. 29, John Wiley \& Sons, 2005.

[3] Troy O McBride, Brian W Pogue, Steven Poplack, Sandra Soho, Wendy A Wells, Shudong Jiang, Keith D Paulsen, et al., "Multispectral near-infrared tomography: a case study in compensating for water and lipid content in hemoglobin imaging of the breast," Journal of biomedical optics, vol. 7, no. 1, pp. 72-79, 2002.

[4] ME Dickinson, G Bearman, S Tille, R Lansford, and SE Fraser, "Multispectral imaging and linear unmixing add a whole new dimension to laser scanning fluorescence microscopy," Biotechniques, vol. 31, no. 6, pp. 1272-1279, 2001.

[5] B Hunt and Olaf Kubler, "Karhunen-loeve multispectral image restoration, part i: Theory," IEEE transactions on acoustics, speech, and signal processing, vol. 32, no. 3, pp. 592-600, 1984.

[6] Nikolas P Galatsanos and Roland T Chin, "Digital restoration of multichannel images," IEEE Transactions on Acoustics, Speech, and Signal Processing, vol. 37, no. 3, pp. 415-421, 1989.

[7] Simon Henrot, Charles Soussen, and David Brie, "Fast positive deconvolution of hyperspectral images," IEEE Transactions on Image Processing, vol. 22, no. 2, pp. 828-833, 2013.

[8] S Bongard, F Soulez, Éric Thiébaut, and É Pecontal, "3d deconvolution of hyper-spectral astronomical data," Monthly Notices of the Royal Astronomical Society, vol. 418, no. 1, pp. 258-270, 2011.

[9] Yingying Song, David Brie, El-Hadi Djermoune, and Simon Henrot, "Regularization parameter estimation for non-negative hyperspectral image deconvolution," IEEE Transactions on Image Processing, vol. 25 , no. 11 , pp. 5316-5330, 2016.

[10] Nikolas P Galatsanos, Aggelos K Katsaggelos, Roland T Chin, and Allen D Hillery, "Least squares restoration of multichannel images," IEEE Transactions on Signal Processing, vol. 39, no. 10, pp. 2222 2236, 1991.

[11] Richard R Schultz and Robert L Stevenson, "Stochastic modeling and estimation of multispectral image data," IEEE Transactions on Image Processing, vol. 4, no. 8, pp. 1109-1119, 1995.

[12] You-Wei Wen, Michael K Ng, and Yu-Mei Huang, "Efficient total variation minimization methods for color image restoration," IEEE Transactions on Image Processing, vol. 17, no. 11, pp. 2081-2088, 2008.

[13] Junfeng Yang, Wotao Yin, Yin Zhang, and Yilun Wang, "A fast algorithm for edge-preserving variational multichannel image restoration," SIAM Journal on Imaging Sciences, vol. 2, no. 2, pp. 569-592, 2009.

[14] MA Hadj-Youcef, François Orieux, Aurélia Fraysse, and Alain Abergel, "Restoration from multispectral blurred data with non-stationary instrument response," in Signal Processing Conference (EUSIPCO), 2017 25th European. IEEE, 2017, pp. 503-507.

[15] Philippe Thévenaz, Thierry Blu, and Michael Unser, "Interpolation revisited [medical images application]," IEEE Transactions on medical imaging, vol. 19, no. 7, pp. 739-758, 2000.

[16] Joseph W Goodman, Introduction to Fourier optics, Roberts and Company Publishers, 2005.

[17] Leonid Yaroslavsky, Digital holography and digital image processing: principles, methods, algorithms, Springer Science \& Business Media, 2013.

[18] Andreï Nikolaevitch Tikhonov, AV Goncharsky, VV Stepanov, and Anatoly G Yagola, Numerical methods for the solution of ill-posed problems, vol. 328, Springer Science \& Business Media, 2013.

[19] Jonathan Richard Shewchuk, "An introduction to the conjugate gradient method without the agonizing pain," 1994.

[20] Patrice Bouchet, Macarena García-Marín, P-O Lagage, Jérome Amiaux, J-L Auguéres, Eva Bauwens, JADL Blommaert, $\mathrm{CH}$ Chen, ÖH Detre, Dan Dicken, et al., "The Mid-Infrared Instrument for the James Webb Space Telescope, III: MIRIM, The MIRI Imager," Publications of the Astronomical Society of the Pacific, vol. 127, no. 953, pp. 612, 2015.

[21] Alistair Glasse, GH Rieke, Eva Bauwens, Macarena García-Marín, ME Ressler, Steffen Rost, Tuomo Ville Tikkanen, Bart Vandenbussche, and GS Wright, "The Mid-Infrared Instrument for the James Webb Space Telescope, IX: Predicted Sensitivity," Publications of the Astronomical Society of the Pacific, vol. 127, no. 953, pp. 686, 2015.
[22] Marshall D Perrin, Rémi Soummer, Erin M Elliott, Matthew D Lallo, and Anand Sivaramakrishnan, "Simulating point spread functions for the James Webb Space Telescope with WebbPSF," International Society for Optics and Photonics, 2012, pp. 84423D-84423D.

[23] Marshall D Perrin, Anand Sivaramakrishnan, Charles-Philippe Lajoie, Erin Elliott, Laurent Pueyo, Swara Ravindranath, and Loïc Albert, "Updated point spread function simulations for jwst with webbpsf," in Space Telescopes and Instrumentation 2014: Optical, Infrared, and Millimeter Wave. International Society for Optics and Photonics, 2014, vol. 9143, p. 91433X.

[24] A Abergel, D Teyssier, JP Bernard, F Boulanger, A Coulais, D Fosse, E Falgarone, M Gerin, M Perault, J-L Puget, et al., "Isocam and molecular observations of the edge of the horsehead nebula," Astronomy \& Astrophysics, vol. 410, no. 2, pp. 577-585, 2003. 